\title{
Recent advances in image treatment for chromosome analysis
}

\author{
A Geneix, P Malet \\ Faculté de Médecine, Laboratoire de Cytogénétique, \\ BP 38, 63000 Clermont-Ferrand Cedex, France \\ (Proceedings of the 9th European Colloquium on Cytogenetics of Domestic Animals; \\ Toulouse-Auzeville, 10-13 July 1990)
}

karyotyping machines / computers / network / image analysis / chromoscan

\section{INTRODUCTION}

Karyotyping machines are now commercially available. More than ten systems have been developed for cytogenetic analysis. The choice of such a system should be based on rigorous criteria, taking into account the user's needs, the purchase cost and the quality of the system.

Evaluation procedures should allow a judicious choice to be made in accordance with the needs and the financial means of the laboratory. In addition to the European procedure, the French ministry submitted the French system to the evaluation of two independent laboratories. We present here the principle criteria retained and, in particular, their application to the system that we have elaborated, the chromoscan. This system is now distributed by the Chromoscan Company.

\section{THE HARDWARE}

\section{Microscope}

The microscope is the first step in the chain of the system. A top quality microscope is required (such as Axio (Zeiss) or Aristo (Leitz)). We have also tested a Nikon microscope with success. It is useless to buy an efficient karyotyping system if the microscope is of poor quality. Some companies provide the microscope with the system to avoid this problem. We think that the choice of the microscope should be made by the biologist after testing. A zoom lens is of great interest for the machine because it allows the image to be adapted to the screen. 


\section{Video camera}

The black and white camera must have good definition $(512 \times 512$ pixels). We think that the high-definition camera is presently too expensive. In addition, if such cameras are used, the system must be adapted to process the increased amount of information. The CCD (charged coupled device) camera is now available for our purpose, but analogic cameras are also suitable. Thomson, Ikegami and Pulnix cameras have been tested with success. The digitalization is made by a matrix card $(1024 \times 1024$ pixels $)$ split into four areas.

\section{Computer}

To avoid excessive purchase and maintenance costs, our preference goes to an efficient compatible personal computer with a hard disk. Computers with 20 megaHertz or more are suitable. This point is particularly important as the more expensive, efficient systems have a specialized computer. The user is, therefore, entirely dependent upon the constructor, who cannot offer the same advantages of cost and updating as those offered to users of personal computers. Chromoscan opted for a PC from the start and other systems, in particular the American ones, have made the same choice. Presently, this judicious choice is becoming generalized.

\section{Network}

A network can connect different work-stations in the same laboratory or situated in different laboratories. All medical and administrative files are available from each station. This system is used in our laboratory. We have also connected our laboratory with a Parisian one using the 'Transcom network'. The transmission is made at the speed of one image per $16 \mathrm{~s}$. Chromoscan was probably the first karyotyping machine which established such a network in Europe.

With the knowledge gained through this experience, we are presently setting up a European network for the exchange of images and the establishment of a cytogenetic library in collaboration with Belgian and Italian research teams.

\section{Storage systems}

Various systems can be used. Our preference goes to the optical disk or the streamer. The images which are stored in these memory banks are digitalized. Therefore, the restitution of the information is perfect, without any of the alterations we observe with analogic systems.

\section{Hard copies}

In order to obtain a paper support, hard copies are proposed. Various systems (thermal, chemical, laser, etc) are available. Often, the systems are expensive and the quality is inferior to the screen image. Therefore, the hard copies are not used for clinical analysis but are kept for the library. Today, the chromoscan hard copies are printed by a Sony-UP 930 printer (fig 1). 

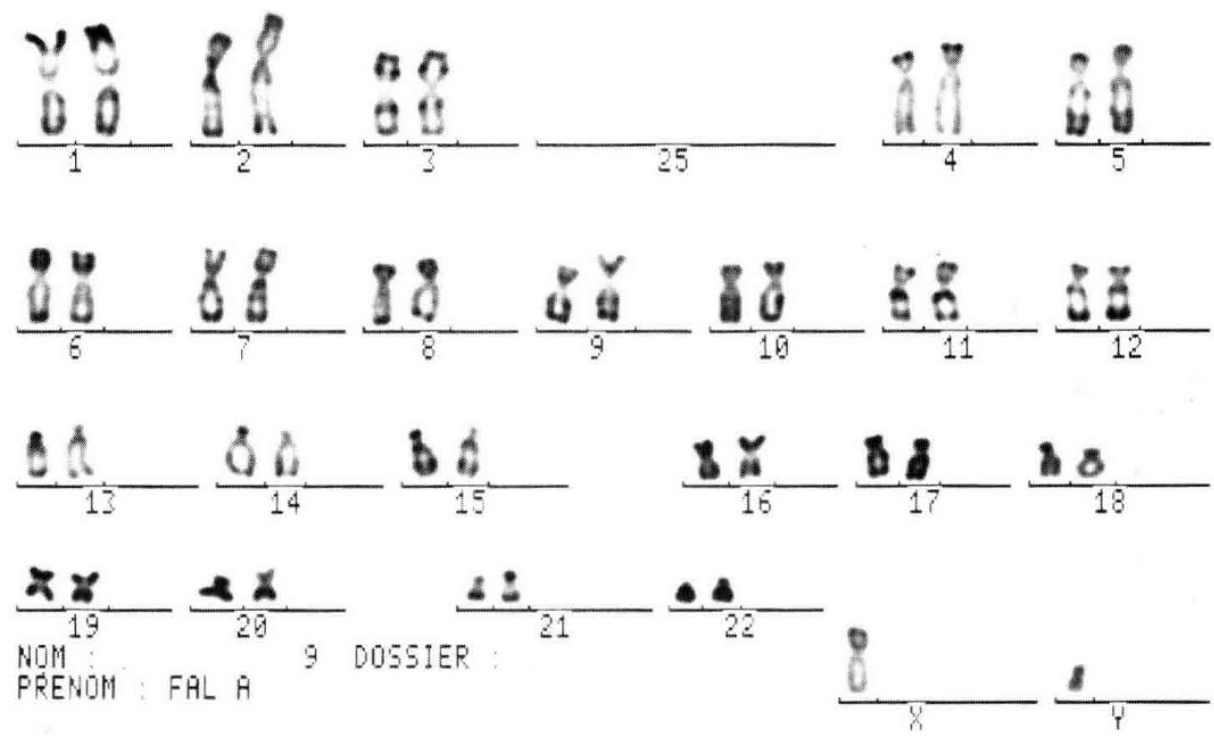

Fig 1. Human fetal karyotype carried out the chromoscan machine. The 25th address is used as a standby position.

\section{THE SOFTWARE}

Several systems propose automatic metaphase location and classification of chromosomes. The studies concern essentially humans and most have been carried out on G-banded preparations. Many investigations have been performed by Danish and British teams (Granum and Thomason, 1990; Lundsteen et al, 1986; Lundsteen and Piper, 1989; Piper and Rutovitz, 1985; Piper and Granum, 1989; Ji, 1989; Sagredo, 1990). The computers are mostly an integrated unit of the system (Magiscan-Cytoscan). Chromoscan (Lundsteen and Piper, 1989; Malet et al, 1989) proposes a system for automatic classification with $R$-banding. In all cases, a preliminary preparation of the image to eliminate artifacts is necessary. The frequency of errors is still excessive. The time wasted rectifying the classification makes the utility of this automation questionable. Its usefulness must be tested separately in each laboratory due to the variability of preparations used.

The interactive classification of chromosomes (possible with chromoscan) is reliable and rapid. It is done following the fully automated extraction of the chromosomes. After classification, each pair of chromosomes can be subjected to a densitometric study, thus enabling the bands to be visualized by the peaks of a curve (fig 2). This is of interest for the detection of aberrations, such as inversions, deletions and translocations.

Specific programs are available for the chromoscan. 


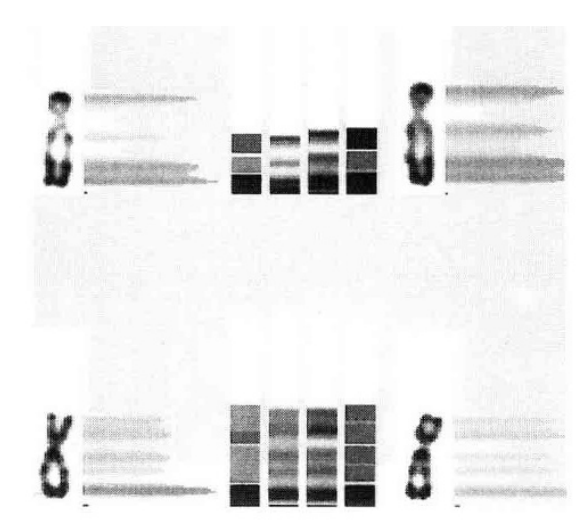

Fig 2. Profile curves. The grey levels are displayed from the top to the bottom of the chromosomes (pairs 5 and 7 in this example). The patterns in the center of the image present the simpler aspects of the chromosomes.

Adaptation of the classification chart

The operator can build the classification frame himself. The method uses an interactive operation. The operator draws the frame with the mouse, like with a pen on paper. The chromoscan machine is therefore suited for karyotyping various species.

\section{Gene mapping}

A special program is available for locating the genes after in situ hybridization with radioactive probes (tables $I$ and $I I$ ).

In order to map the genes, in situ hybridization is usually followed by a statistical study of the distribution of the silver grains. This method is long and tedious. We cannot distinguish one high signal from another with a statistical test of frequencies (table I). Each grain has the same value, even in cases of multicopy genes.

We have drawn up a flowchart for the quantification of the levels of grey intensity using the chromoscan machine. The intensity of the radioactive signal is evaluated according to the sum of the values assigned to all the grey intensity levels in each marked area. Each chromosome band in every mitosis is thus measured. For each band, the average value and the statistical variation appear numerically. The $\chi^{2}$ test for the frequencies of the signal and Student's $t$ test for the averages of the intensities are applied to reveal the most probable locus of the gene (table II).

For example, we tested a multicopy gene and the locus appeared clearly near the chromosome no 3 centromere (tables I and II). In this case, Student's $t$ test (calculated with few data) shows a significant difference $(\alpha<1 \%)$ between 03p00 and the band that is nearest in intensity at $02 \mathrm{p} 00$. The $\chi^{2}$ test on the frequencies shows an even more significant difference $\left(\alpha<1^{\circ} /^{\circ}\right)$ and this for every band compared with 03 p00. 
Tables I and II. In situ hybridization results. The 03 p00 band (centromeric band) presents the strongest intensity.

Table I.

\begin{tabular}{ccr}
\hline Mitotic number & Band number & Intensity \\
\hline 1 & $03 \mathrm{p} 00$ & 5608 \\
1 & $12 \mathrm{p} 00$ & 1680 \\
1 & $03 \mathrm{q} 28$ & 716 \\
1 & $03 \mathrm{p} 00$ & 476 \\
2 & $03 \mathrm{p} 00$ & 8827 \\
2 & $09 \mathrm{p} 00$ & 3565 \\
3 & $03 \mathrm{p} 00$ & 3064 \\
3 & $12 \mathrm{q} 24$ & 1152 \\
3 & $17 \mathrm{p} 11$ & 742 \\
4 & $03 \mathrm{p} 00$ & 5381 \\
4 & $16 \mathrm{p} 11$ & 1268 \\
4 & $07 \mathrm{p} 00$ & 933 \\
4 & $05 \mathrm{p} 14$ & 510 \\
5 & $02 \mathrm{p} 00$ & 5308 \\
5 & $03 \mathrm{q} 26$ & 1264 \\
5 & $03 \mathrm{p} 00$ & 1106 \\
5 & $0 \mathrm{xp} 21$ & 250 \\
6 & $03 \mathrm{p} 00$ & 13152 \\
6 & $05 \mathrm{p} 14$ & 1233 \\
6 & $04 \mathrm{p} 11$ & 494 \\
7 & $03 \mathrm{p} 00$ & 9071 \\
7 & $11 \mathrm{p} 15$ & 1763 \\
7 & $09 \mathrm{p} 33$ & 762 \\
7 & $11 \mathrm{p} 11$ & 506 \\
\hline
\end{tabular}

Table II.

\begin{tabular}{ccc}
\hline Band number & Average intensity & Variance $\times 10$ \\
\hline $03 \mathrm{p} 00$ & 6587 & 1.4845 \\
$12 \mathrm{p} 00$ & 240 & 0.0308 \\
$03 \mathrm{q} 28$ & 102 & 0.0056 \\
$06 \mathrm{p} 00$ & 68 & 0.0024 \\
$09 \mathrm{p} 00$ & 509 & 0.1390 \\
$12 \mathrm{q} 24$ & 165 & 0.0145 \\
$17 \mathrm{p} 11$ & 106 & 0.0060 \\
$16 \mathrm{p} 11$ & 181 & 0.0176 \\
$07 \mathrm{p} 00$ & 133 & 0.0095 \\
$05 \mathrm{p} 14$ & 249 & 0.0159 \\
$02 \mathrm{p} 00$ & 758 & 0.3082 \\
$03 \mathrm{q} 26$ & 181 & 0.0174 \\
$0 \mathrm{Xq} 21$ & 36 & 0.0006 \\
$04 \mathrm{p} 11$ & 71 & 0.0026 \\
$11 \mathrm{p} 15$ & 255 & 0.0348 \\
$09 \mathrm{p} 23$ & 109 & 0.0063 \\
$11 \mathrm{p} 11$ & 72 & 0.0028 \\
\hline
\end{tabular}


This variation is due, first of all, to the fact that the $\chi^{2}$ test is very effective; secondly, the grain count alone does not take the size of the grains into account, and this is reflected in the results.

In cases like the one above, where both tests are significant, they both lead to the same conclusion: the locus is probably at $03 \mathrm{p} 00$. But there can be cases in which the conclusion is less obvious: Student's $t$ test is significant but the $\chi^{2}$ test is not or vice versa. In the first case, this indicates that one or several loci might be more frequent than the locus that is globally the most marked. The decision made must be prudent, taking into account the nature of the probes: repetitive or single-copy.

If the $\chi^{2}$ test is significant and Student's $t$ test is not, this indicates that the most frequent locus is not globally the most marked. In a case like this, it seems to us that only an intense background noise can explain the result. Caution must be used in making this judgement.

Our example has an obvious result, because the probe is a multicopy gene probe. In other cases, the use of our work-station is very helpful.

In conclusion, we find it desirable to perform both tests. Since the system is automated, these two operations are carried out without difficulty.

\section{Chromosome number distribution}

After the number of chromosomes is counted for many metaphase cells, the histogram of the distribution is displayed and the modal number is calculated. The operations are performed automatically. The modal number is of great interest in cancer cytogenetics.

\section{CONCLUSION}

Instead of a large powerful system, efficient but expensive, we have opted to elaborate a system based on the use of an easily updated microcomputer of reasonable price. The addition of complementary hardware (arithmetic co-processor, network, hardcopies) has allowed chromoscan to be the precursor of the efficient and easy use of the latest developments in microcomputing. Tested in more than ten laboratories, it has shown itself to be well adapted to needs in cytogenetics. An annual meeting of all the users of this system enables us to take stock of progress made and to fix future objectives.

\section{ACKNOWLEDGMENTS}

The European Community (Concerted Action in Automatism of Cytogenetics - CAAC); The Chromoscan Company (Montpellier, France); ANVAR (national support); Auvergne University (France).

\section{REFERENCES}

Granum E, Thomason MG (1990) Automatically inferred Markov network. Models for classification of chromosomal band pattern structures. Cytometry 11, 26-39 
Ji L (1989) Intelligent splitting in the chromosome domain. Pattern Recognition 22, 519532

Lundsteen C, Piper J (1989) Automation of Cytogenetics. Springer Verlag

Lundsteen C, Gerdes T, Maahr J (1986) Automatic classification of chromosomes as part of a routine system for clinical analysis. Cytometry $7,1-7$

Malet P, Geneix A, Bonton P, Grouche L, Perissel B (1989) L'analyse chromosomique par traitement d'images : aspects récents et perspectives. Ann Génét 32, 164-168

Piper J, Rutovitz D (1985) Data structures for image processing in a C language and unix environment. Pattern Recognition Lett 3, 119-129

Piper J, Granum E (1989) On fully automatic feature measurement for banded chromosome classification. Cytometry 10, 242-245

Sagredo G (1990) Abstracts of the 11th European Workshop on Automated Cytogenetics. Genetica Medica, Hospital Ramon Cajal, Madrid 